\title{
ESTIMATION OF GENETIC PARAMETERS IN THE ANALYSIS OF SQUARE LATTICE EXPERIMENT GROUP(1)
}

\author{
JOSÉ MARCELO SORIANO VIANA (2) \& ADAIR JOSÉ REGAZZI (3)
}

\begin{abstract}
Aiming to demonstrate how to obtain unbiased estimates of genetic parameters of base populations, unaffected by genotype $\mathrm{x}$ environment effects, this paper presents the variance and covariance components of the intra-block analysis of a group of square lattice experiments and the estimators of the components associated to treatment effect. Random model and mixed models with environment effect fixed and other effects random are considered. In the analysis with treatments not corrected for blocks/replications/environments, the estimators of the variance and covariance components due to treatment effect are different from those of the analysis considering the complete block model. Data from two experiments of a breeding program of Eucalyptus pyrocarpa were used for genetic analysis. The analysis of variance of height and diameter indicated absence of interaction between progeny and environment. Due to this result, the prediction of the direct and indirect genetic gains was based on the mean of the two environments. The high estimates of narrow sense heritabilities and additive genetic correlation indicate that selection of the superior families will be effective in changing the means of the base population for both traits.
\end{abstract}

Index terms: quantitative genetics, genetic parameters, variance components, covariance components, joint analysis, square lattice.

\footnotetext{
(1) Received for publication in April 23, 1997, and approved in December 9, 1998.

(2) Engenheiro Agrônomo D.Sc., Departamento de Biologia Geral, Universidade Federal de Viçosa, 36.570-000 Viçosa (MG).

(3) Eng.-Agr., D.Sc., Departamento de Informática, Universidade Federal de Viçosa, 36.570-000 Viçosa (MG).
} 


\title{
RESUMO
}

\section{ESTIMAÇÃO DE PARÂMETROS GENÉTICOS NA ANÁLISE DE GRUPO DE EXPERIMENTOS EM LÁTICE QUADRADO}

\begin{abstract}
Neste trabalho, discute-se a estimação de parâmetros genéticos de populações-base, quando as famílias amostradas foram avaliadas em dois ou mais ambientes, no delineamento em látice quadrado. Na parte teórica, são apresentados os componentes de variância e covariância da análise intrablocos de grupo de experimentos em látice quadrado e os estimadores dos componentes associados a efeito de tratamento, considerando estimação pelo método dos quadrados mínimos ordinário. Os estimadores dos componentes da variância e covariância da análise com tratamentos não ajustados diferem dos da análise segundo modelo em blocos completos. Além de modelo aleatório, consideram-se também os mistos com efeito de ambiente fixo e demais efeitos aleatórios. Dados de dois experimentos de um programa de melhoramento de Eucalyptus pyrocarpa foram usados para análise genética. Como em relação às características altura e diâmetro não houve evidência de interação progênie $\mathrm{x}$ ambiente, a predição de ganhos diretos e indiretos foi feita com base na média dos dois ambientes. Os valores elevados da herdabilidade em sentido restrito e da correlação genética aditiva evidenciam que a seleção das famílias superiores será eficiente em alterar as médias da população-base, para as duas características.
\end{abstract}

Termos de indexação: genética quantitativa, parâmetros genéticos, componentes da variância, componentes da covariância, análise conjunta, látice quadrado.

\section{INTRODUCTION}

The evaluation of a group of treatments (families, varieties etc.) in more than one environmental condition is common in breeding programs. This allows to study the interaction between treatments and environments (local and/or years, and so on) and, when the treatments are families sampled from a base population, the estimation of genetic parameters not affected by the progeny x environment interaction. Due to its implication on the selective process by decreasing the correlation between phenotypic and genotypic values, the genotype $\mathrm{x}$ environment interaction is a complex and widely investigated problem, which must be considered in breeding programs. Since a high number of treatments is normally used in these experiments, the lattice design has been frequently used (Zuber, 1942; Johnson \& Murphy, 1943; Torrie et al., 1943; Wellhausen, 1943; Bancroft \& Smith, 1949; Sahagun-Castellanos \& Frey, 1990; Beninati \& Busch, 1992; Chaves \& Miranda Filho, 1992; Singh et al, 1992; Arriel et al., 1993; Lin et al., 1993; Michelini
\& Hallauer, 1993; Moncada et al., 1993; Oliveira, 1993; Ferrão et al., 1994, and Rezende \& Ramalho, 1994), contributing to increase experimental error control efficiency (Cochran \& Cox, 1957, and Federer, 1955).

In many cases, however, the joint analysis of lattice experiments and, particularly, the estimation of the variance and covariance components used to estimate genetic parameters, e.g., genotypic variance between families, additive genetic variance, heritability on a family mean basis, genotypic correlation and expected genetic gain (Kempthorne, 1957), involve approximate processes. The lattice design sometimes is not taken into account with the complete block model being considered.

An exact estimation of the variance and covariance components is possible if the expected mean squares are known or if the program used for the analysis makes itself the estimation (for example, the VARCOMP procedure of SAS/STAT ${ }^{\circledR}$ (SAS Institute, 1989)). The purpose of this paper is to show how to estimate genetic parameters of populations, using the 
estimates of the variance and covariance components of the intra-block analysis of a group of square lattice experiments, considering the least squares method.

\section{THE INTRA-BLOCK ANALYSIS OF A GROUP OF SQUARE LATTICE EXPERIMENTS}

The complete statistical model is:

$+\mathrm{e}_{\mathrm{il}(\mathrm{j})(\mathrm{g})} \mathrm{Y}_{\mathrm{il}(\mathrm{j})(\mathrm{g})}=\mu+\mathrm{t}_{\mathrm{i}}+(\mathrm{r} \mid \mathrm{a})_{\mathrm{j}(\mathrm{g})}+(\mathrm{b}|\mathrm{r}| \mathrm{a})_{1(\mathrm{j})(\mathrm{g})}+\mathrm{a}_{\mathrm{g}}+(\mathrm{ta})_{\mathrm{ig}}$ where:

$\mathrm{Y}_{\mathrm{il}(\mathrm{j})(\mathrm{g})}$ is the observation of the treatment $\mathrm{i}(\mathrm{i}=1, \ldots, \mathrm{v}$ $\left.=\mathrm{k}^{2}\right)$ in the block $1(1=1, \ldots, \mathrm{k})$ of the replication $\mathrm{j}(\mathrm{j}=$ $1, \ldots, \mathrm{m})$, in the environment $\mathrm{g}(\mathrm{g}=1, \ldots, \mathrm{s})$;

$\mu$ is a constant common to all observations;

$t_{i}$ is the effect of the treatment $i$;

$(r \mid a)_{j(g)}$ is the effect of the replication $j$ in the environment $\mathrm{g}$;

$(b|r| a)_{1(j)(g)}$ is the effect of the block 1 of the replication $\mathrm{j}$, in the environment $\mathrm{g}$;

$\mathrm{a}_{\mathrm{g}}$ is the effect of the environment $\mathrm{g}$;

$(\mathrm{ta})_{\mathrm{ig}}$ is the effect of the interaction between the treatment $i$ and the environment $\mathrm{g}$;

$\mathrm{e}_{\mathrm{il}(\mathrm{j})(\mathrm{g})}$ is the error associated to the observation $\mathrm{Y}_{\mathrm{il}(\mathrm{j})(\mathrm{g})}$; $\mathrm{e}_{\mathrm{i}(\mathrm{j})(\mathrm{g})} \sim \mathrm{N}\left(0, \sigma^{2}\right)$, independent.

The matricial form of the linear model we consider is:

$\mathrm{Y}=\mathrm{X} \Theta+\mathrm{e} ; \varepsilon \sim \mathrm{N}\left(\Phi, \sigma^{2} \mathrm{I}\right)$

with:

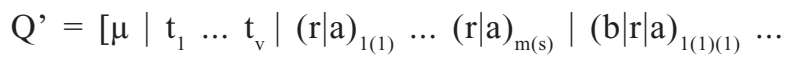
$\left.(\mathrm{b}|\mathrm{r}| \mathrm{a})_{\mathrm{k}(\mathrm{m})(\mathrm{s})}\left|\mathrm{a}_{1} \ldots \mathrm{a}_{\mathrm{s}}\right|(\mathrm{ta})_{11} \ldots(\mathrm{ta})_{\mathrm{vs}}\right]$

$=\left[\mu\left|\tau^{\prime}\right| \alpha^{\prime}\left|\beta^{\prime}\right| \delta^{\prime} \mid \tau \delta^{\prime}\right]$

In the analyses of variance the orthogonal partitions of the reduction in the total sum of squares due to fitting the complete model will be as follows: $\mathrm{R}(\mu, \tau, \alpha, \beta, \delta, \tau \delta)=\mathrm{R}(\mu)+\mathrm{R}(\delta \mid \mu)+\mathrm{R}(\alpha \mid \mu, \delta)+\mathrm{R}(\beta \mid \mu$, $\delta, \alpha)+\mathrm{R}(\tau \mid \mu, \delta, \alpha, \beta)+\mathrm{R}(\tau \delta \mid \mu, \tau, \delta, \alpha, \beta)=\mathrm{R}(\mu)+$ $\mathrm{R}(\delta \mid \mu)+\mathrm{R}(\alpha \mid \mu, \delta)+\mathrm{R}(\tau \mid \mu, \delta, \alpha)+\mathrm{R}(\beta \mid \mu, \tau, \delta, \alpha)+$ $\mathrm{R}(\tau \delta \mid \mu, \tau, \delta, \alpha, \beta)$

where $\mathrm{R}()=.\mathrm{Y}^{\prime} \mathrm{X}\left(\mathrm{X}^{\prime} \mathrm{X}\right)^{\mathrm{G}} \mathrm{X}^{\prime} \mathrm{Y}$ is the reduction in the total sum of squares due to fitting a certain model, with rank of $X\left(X^{\prime} X\right)^{G} X^{\prime}=$ rank of $X$ degrees of freedom, being $\left(X^{\prime} X\right)^{G}$ any generalized inverse of $X^{\prime} X$, and $R(. \mid$.$) is a difference between two R($.$) terms$ (Searle, 1971, 1992, and Graybill, 1976).

The analyses of variance related to the two partitions of $\mathrm{R}(\mu, \tau, \alpha, \beta, \delta, \tau \delta)$ are shown in Table 1 .

\subsection{The intra-block analysis of random model}

The assumptions of the statistical model are:

a) $\mathrm{t}_{\mathrm{i}} \sim \mathrm{N}\left(0, \sigma_{\mathrm{t}}^{2}\right)$, independent;

b) $(\mathrm{r} \mid \mathrm{a})_{\mathrm{j}(\mathrm{g})} \sim \mathrm{N}\left(0, \sigma_{\mathrm{r}}^{2}\right)$, independent;

c) $(\mathrm{b}|\mathrm{r}| \mathrm{a})_{1(\mathrm{j})(\mathrm{g})} \sim \mathrm{N}\left(0, \sigma_{\mathrm{b}}^{2}\right)$, independent;

d) $\mathrm{a}_{\mathrm{g}} \sim \mathrm{N}\left(0, \sigma_{\mathrm{a}}^{2}\right)$, independent;

e) (ta) $)_{\mathrm{ig}} \sim \mathrm{N}\left(0, \sigma_{\mathrm{ta}}^{2}\right)$, independent;

f) $\mathrm{e}_{\mathrm{il}(\mathrm{j})(\mathrm{g})} \sim \mathrm{N}\left(0, \sigma^{2}\right)$, independent;

g) $t_{i},(r \mid a)_{j(g)},(b|r| a)_{(j)(g)}, a_{g},(t a)_{i g}$ and $e_{i l(j)(g)}$ are independent.

In the covariance matrix of $\mathrm{Y}, \operatorname{Cov}(\mathrm{Y})=\mathrm{E}\{[\mathrm{Y}$ $\left.\mathrm{E}(\mathrm{Y})][\mathrm{Y}-\mathrm{E}(\mathrm{Y})]^{\prime}\right\}=\Sigma_{(\mathrm{n})} \cdot \sigma^{2} \mathrm{I}, \mathrm{n}=\mathrm{smk}^{2}$, the elements are:

$\mathrm{V}\left(\mathrm{Y}_{\mathrm{i}(\mathrm{l})(\mathrm{g})}\right)=\sigma_{\mathrm{t}}^{2}+\sigma_{\mathrm{r}}^{2}+\sigma_{\mathrm{b}}^{2}+\sigma_{\mathrm{a}}^{2}+\sigma_{\mathrm{ta}}^{2}+\sigma^{2}=\mathrm{C}_{0}$

$\operatorname{Cov}\left(\mathrm{Y}_{\mathrm{i}(\mathrm{j})(\mathrm{g} g}, \mathrm{Y}_{\mathrm{i}^{\prime} \mathrm{l}^{(\mathrm{j})(\mathrm{g})}}\right)=\sigma_{\mathrm{r}}^{2}+\sigma_{{ }_{\mathrm{b}}}^{2}+\sigma_{\mathrm{a}}^{2}=\mathrm{C}_{1} \quad\left(\mathrm{i} \cdot \mathrm{i}^{\prime}\right)$

$\operatorname{Cov}\left(\mathrm{Y}_{\mathrm{i}(\mathrm{j})(\mathrm{g})}, \mathrm{Y}_{\mathrm{i}^{\prime}{ }^{\prime}(\mathrm{j})(\mathrm{g})}\right)=\sigma_{\mathrm{r}}^{2}+\sigma_{\mathrm{a}}^{2}=\mathrm{C}_{2} \quad\left(\mathrm{i} \bullet \mathrm{i} \mathrm{i}^{\prime}\right.$ and $\left.1 \cdot \mathrm{l}^{\prime}\right)$

$\operatorname{Cov}\left(\mathrm{Y}_{\mathrm{i}(\mathrm{j})(\mathrm{g})}, \mathrm{Y}_{\mathrm{i}{ }^{\prime}\left(\mathrm{j}^{\prime}\right)(\mathrm{g})}\right)=\sigma_{\mathrm{t}}^{2}+\sigma_{\mathrm{a}}^{2}+\sigma_{\mathrm{ta}}^{2}=\mathrm{C}_{3} \quad\left(\mathrm{j} \cdot \mathrm{j}^{\prime}\right)$

$\operatorname{Cov}\left(Y_{i 1(j)(g)}, Y_{i^{\prime} l^{\prime}\left(j^{\prime}\right)(g)}\right)=\sigma_{a}^{2}=C_{4} \quad\left(i \cdot i^{\prime}\right.$ and $\left.j \cdot j^{\prime}\right)$

$\operatorname{Cov}\left(\mathrm{Y}_{\mathrm{il}(\mathrm{j})(\mathrm{g})}, \mathrm{Y}_{\mathrm{il}{ }^{\prime}\left(\mathrm{j}^{\prime}\right)\left(\mathrm{g}^{\prime}\right)}\right)=\sigma_{\mathrm{t}}^{2}=\mathrm{C}_{5} \quad\left(\mathrm{~g} \cdot \mathrm{g}^{\prime}\right)$

$\operatorname{Cov}\left(\mathrm{Y}_{\mathrm{i}(\mathrm{l}(\mathrm{j})(\mathrm{g})}, \mathrm{Y}_{\mathrm{i}^{\prime}{ }^{\prime} \mathrm{j}^{\prime}\left(\mathrm{j}^{\prime}\right)\left(\mathrm{g}^{\prime}\right)}\right)=0=\mathrm{C}_{6} \quad\left(\mathrm{i} \cdot \mathrm{i}^{\prime}\right.$ and $\left.\mathrm{g} \cdot \mathrm{g} \mathrm{g}^{\prime}\right)$

Using the property of mathematical expectation of quadratic forms (Searle, 1971, Searle et al. 1992, and Graybill, 1976), the expected values below and those presented in Table 2 can be demonstrated:

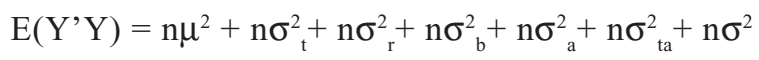
$\mathrm{E}[\mathrm{R}(\mu)]=\mathrm{n} \mu^{2}+\mathrm{ms} \sigma_{\mathrm{t}}^{2}+\mathrm{v} \sigma_{\mathrm{r}}^{2}+\mathrm{k} \sigma_{\mathrm{b}}^{2}+\mathrm{mv} \sigma_{\mathrm{a}}^{2}+\mathrm{m} \sigma_{\mathrm{ta}}^{2}+\sigma^{2}$ $\mathrm{E}[\mathrm{R}(\mu, \tau, \delta, \alpha, \beta)]=n \mu^{2}+n \sigma_{\mathrm{t}}^{2}+\mathrm{n}{\sigma_{\mathrm{r}}^{2}}_{\mathrm{r}}+\mathrm{n}{\sigma^{2}}_{\mathrm{b}}+\mathrm{n}{\sigma^{2}}_{\mathrm{a}}+$ $[\mathrm{mks}+\mathrm{mk}(\mathrm{k}-1)]{\sigma_{\mathrm{ta}}^{2}}^{2}+(\mathrm{v}+\mathrm{mks}-1) \sigma^{2}$

Considering that the treatments are families sampled from a base population, the two estimators of the variance of the genotypic means of the progenies that can be obtained from the reference population $\left(\sigma_{t}^{2}\right)$ are: 


$$
\begin{aligned}
& \hat{\sigma}_{\mathrm{t}(1)}^{2}=\left(\frac{\mathrm{k}+1}{\mathrm{k}}\right)\left[\frac{\mathrm{MST} \text { (Adj.) }-\mathrm{MSTE}}{\mathrm{ms}}\right] \\
& \hat{\sigma}_{\mathrm{t}(2)}^{2}=\left[\frac{\mathrm{MST}(\mathrm{N} \cdot \mathrm{Adj} .)-\mathrm{MSe}-\mathrm{m} \hat{\sigma}_{\mathrm{ta}}^{2}-\left(\frac{\mathrm{k}}{\mathrm{k}+1}\right) \hat{\sigma}_{\mathrm{b}(2)}^{2}}{\mathrm{~ms}}\right] \neq\left[\frac{\mathrm{MST}(\mathrm{N} \cdot \mathrm{Adj} .)-\mathrm{MSTE}_{\mathrm{CB}}}{\mathrm{ms}}\right]
\end{aligned}
$$

where:

$$
\begin{aligned}
& \hat{\sigma}_{\mathrm{ta}}^{2}=\left(\frac{\mathrm{k}+1}{\mathrm{k}}\right)\left(\frac{\mathrm{MSTE}-\mathrm{MSe}}{\mathrm{m}}\right) \\
& \left.\hat{\sigma}_{\mathrm{b}(2)}^{2}=\left[\frac{\mathrm{ms}}{\mathrm{k}(\mathrm{ms}-1)}\right][\mathrm{MSB} \nmid \mathrm{R}\} \mathrm{E}(\mathrm{Adj} .)-\mathrm{MSe}-\left(\frac{\mathrm{S}-1}{\mathrm{~s}}\right) \hat{\sigma}_{\mathrm{ta}}^{2}\right]
\end{aligned}
$$

and $\mathrm{MSTE}_{\mathrm{CB}}$ is the treatments $\mathrm{x}$ environments interaction mean square, considering the complete block model.

Therefore, $\hat{\sigma}_{t(2)}^{2}$ is not the estimator of $\sigma_{t}^{2}$ of the analysis according to the complete block model.

The following statistical models are considered to estimate covariance components:

$$
\begin{aligned}
& \mathrm{Y}_{\mathrm{i}(\mathrm{j})(\mathrm{g})}=\mu_{\mathrm{Y}}+\mathrm{t}_{\mathrm{iY}}+(\mathrm{r} \mid \mathrm{a})_{\mathrm{j}(\mathrm{g}) \mathrm{Y}}+(\mathrm{b}|\mathrm{r}| \mathrm{a})_{\mathrm{I}(j)(\mathrm{g}) \mathrm{Y}}+\mathrm{a}_{\mathrm{gY}}+(\mathrm{ta})_{\mathrm{igY}}+ \\
& +\mathrm{e}_{\mathrm{il}(\mathrm{j})(\mathrm{g}) \mathrm{Y}} \\
& \mathrm{X}_{\mathrm{i}(\mathrm{j})(\mathrm{g})}=\mu_{\mathrm{X}}+\mathrm{t}_{\mathrm{iX}}+(\mathrm{r} \mid \mathrm{a})_{\mathrm{j}(\mathrm{g}) \mathrm{X}}+(\mathrm{b}|\mathrm{r}| \mathrm{a})_{\mathrm{I}(\mathrm{j})(\mathrm{g}) \mathrm{X}}+\mathrm{a}_{\mathrm{gX}}+(\mathrm{ta})_{\mathrm{igX}}+ \\
& +\mathrm{e}_{\mathrm{il}(\mathrm{j})(\mathrm{g}) \mathrm{X}} \\
& \mathrm{Y}_{\mathrm{i}(\mathrm{j})(\mathrm{g})}+\mathrm{X}_{\mathrm{i} 1 \mathrm{j}(\mathrm{g})}=\left(\mu_{\mathrm{Y}}+\mu_{\mathrm{X}}\right)+\left(\mathrm{t}_{\mathrm{iY}}+\mathrm{t}_{\mathrm{iX}}\right)+\left[(\mathrm{r} \mid \mathrm{a})_{\mathrm{j}(\mathrm{g}) \mathrm{Y}}+\right. \\
& \left.(r \mid a)_{j(g) X}\right]+\left[(b|r| a)_{1(j)(g) Y}+(b|r| a)_{1(j)(g) X}\right]+\left(a_{g Y}+a_{g X}\right)+ \\
& {\left[(\mathrm{ta})_{\mathrm{igY}}+(\mathrm{ta})_{\mathrm{igX}}\right]+\left(\mathrm{e}_{\mathrm{il}(\mathrm{j})(\mathrm{g}) \mathrm{Y}}+\mathrm{e}_{\mathrm{i}(\mathrm{l})(\mathrm{g}) \mathrm{X}}\right)} \\
& =\mu+\mathrm{t}_{\mathrm{i}}+(\mathrm{r} \mid \mathrm{a})_{\mathrm{j}(\mathrm{g})}+(\mathrm{b}|\mathrm{r}| \mathrm{a})_{1(\mathrm{j})(\mathrm{g})}+\mathrm{a}_{\mathrm{g}}+(\mathrm{ta})_{\mathrm{ig}}+\mathrm{e}_{\mathrm{i}(\mathrm{j})(\mathrm{g})}
\end{aligned}
$$

where $\mathrm{Y}$ and $\mathrm{X}$ are random variables.

Let us consider random models and the following assumptions: (a) $t_{\mathrm{i}}=\left(\mathrm{t}_{\mathrm{iY}}+\mathrm{t}_{\mathrm{iX}}\right) \sim\left(0, \sigma_{\mathrm{t}}^{2}=\sigma_{\mathrm{tY}}^{2}+\sigma_{\mathrm{tX}}^{2}+2 \sigma_{\mathrm{tYX}}\right)$, independent;

(b) $(\mathrm{r} \mid \mathrm{a})_{\mathrm{j}(\mathrm{g})}=\left[(\mathrm{r} \mid \mathrm{a})_{\mathrm{j}(\mathrm{g}) \mathrm{Y}}+(\mathrm{r} \mid \mathrm{a})_{\mathrm{j}(\mathrm{g}) \mathrm{X}}\right] \sim\left(0, \sigma_{\mathrm{r}}^{2}=\sigma_{\mathrm{rY}}^{2}+\sigma_{\mathrm{rX}}^{2}\right.$ $\left.+2 \sigma_{\mathrm{rYX}}\right)$, independent;

(c) $(\mathrm{b}|\mathrm{r}| \mathrm{a})_{{ }_{(j)(\mathrm{g})}}=\left[(\mathrm{b}|\mathrm{r}| \mathrm{a})_{1(j)(\mathrm{g}) \mathrm{Y}}+(\mathrm{b}|\mathrm{r}| \mathrm{a})_{\mathrm{Ij}_{(j)(g) X}}\right] \sim\left(0, \sigma_{\mathrm{b}}^{2}=\right.$ $\left.=\sigma_{\mathrm{bY}}^{2}+\sigma_{\mathrm{bX}}^{2}+2 \sigma_{\mathrm{bYX}}\right)$, independent;

(d) $\mathrm{a}_{\mathrm{g}}=\left(\mathrm{a}_{\mathrm{gY}}+\mathrm{a}_{\mathrm{gX}}\right) \sim\left(0, \sigma_{\mathrm{a}}^{2}=\sigma_{\mathrm{aY}}^{2}+\sigma_{\mathrm{aX}}^{2}+2 \sigma_{\mathrm{aYX}}\right)$, independent;

(e) $(\mathrm{ta})_{\mathrm{ig}}=\left[(\mathrm{ta})_{\mathrm{igY}}+(\mathrm{ta})_{\mathrm{igX}}\right] \sim\left(0, \sigma_{\mathrm{ta}}^{2}=\sigma_{\mathrm{taY}}^{2}+\sigma_{\mathrm{taX}}^{2}+\right.$ $\left.2 \sigma_{\text {taYX }}\right)$, independent;

(f) $\mathrm{e}_{\mathrm{il}(\mathrm{j})(\mathrm{g})}=\left[\mathrm{e}_{\mathrm{i}(\mathrm{j})(\mathrm{g}) \mathrm{Y}}+\mathrm{e}_{\mathrm{il}(\mathrm{j})(\mathrm{g}) \mathrm{X}}\right] \sim\left(0, \sigma^{2}=\sigma_{\mathrm{Y}}^{2}+\sigma^{2}{ }_{\mathrm{X}}+\right.$ $\left.+2 \sigma_{\mathrm{YX}}\right)$, independent;

$(g) t_{i},(r \mid a)_{j(g)},(b|r| a)_{1(j)(g)}, a_{g},(t a)_{i g}$ and $e_{i 1(j)(g)}$ are independent.

From previous results, the expected mean squares presented in Table 3 are obtained. The two estimators of the covariance between genotypic means of the same family, in relation to $\mathrm{Y}$ and $\mathrm{X}\left(\sigma_{\mathrm{tYX}}\right)$, are: 


$$
\begin{aligned}
& \hat{\sigma}_{\mathrm{tYX}(1)}=\left(\frac{\mathrm{k}+1}{\mathrm{k}}\right)\left\{\frac{[\operatorname{MST}(\operatorname{Adj} .)(\mathrm{Y}+\mathrm{X})-\operatorname{MST}(\operatorname{Adj} .)(\mathrm{Y})-\operatorname{MST}(\operatorname{Adj} .)(\mathrm{X})]-[\operatorname{MSTE}(\mathrm{Y}+\mathrm{X})-\operatorname{MSTE}(\mathrm{Y})-\operatorname{MSTE}(\mathrm{X})]}{2 \mathrm{~ms}}\right\} \\
& \hat{\sigma}_{\mathrm{tYX}(2)}=\left[\frac{\operatorname{MST}(\mathrm{N} \cdot A d j .)(\mathrm{Y}+\mathrm{X})-\operatorname{MST}(\mathrm{N} \cdot \operatorname{Adj} .)(\mathrm{Y})-\mathrm{MST}(\mathrm{N} . \text { Adj. })(\mathrm{X})-2 \hat{\sigma}_{\mathrm{YX}}-2 \mathrm{~m} \hat{\sigma}_{\mathrm{taYX}}-2\left(\frac{\mathrm{k}}{\mathrm{k}+1}\right) \hat{\sigma}_{\mathrm{bYX}(2)}}{2 \mathrm{~ms}}\right]
\end{aligned}
$$

where:

$$
\begin{aligned}
& \hat{\sigma}_{\mathrm{YX}}=\frac{\operatorname{MSe}(\mathrm{Y}+\mathrm{X})-\operatorname{MSe}(\mathrm{Y})-\operatorname{MSe}(\mathrm{X})}{2} \\
& \hat{\sigma}_{\mathrm{taYX}}=\left(\frac{\mathrm{k}+1}{\mathrm{k}}\right)\left(\frac{\operatorname{MSTE}(\mathrm{Y}+\mathrm{X})-\operatorname{MSTE}(\mathrm{Y})-\operatorname{MSTE}(\mathrm{X})-2 \sigma_{\mathrm{YX}}}{2 \mathrm{~m}}\right)
\end{aligned}
$$$$
\left.\left.\left.\left.\left.\left.\hat{\sigma}_{\mathrm{bYX}(2)}=\left[\frac{\mathrm{ms}}{2 \mathrm{k}(\mathrm{ms}-1)}\right][\mathrm{MSB}\} \mathrm{R}\right\} \mathrm{E}(\operatorname{Adj} .)(\mathrm{Y}+\mathrm{X})-\mathrm{MSB}\right\} \mathrm{R}\right\} \mathrm{E}(\operatorname{Adj} .)(\mathrm{Y})-\mathrm{MSB}\right\} \mathrm{R}\right\} \mathrm{E}(\operatorname{Adj} .)(\mathrm{X})-2 \sigma_{\mathrm{YX}}-2\left(\frac{\mathrm{S}-1}{\mathrm{~s}}\right) \hat{\sigma}_{\mathrm{taYX}}\right]
$$

\subsection{The intra-block analysis of thw mixed model with environment effect fixed and other effects random}

If the number of environments is reduced they cannot be a representative sample of a population. In these cases and when the researcher is interested in inferring about the chosen environments, their effects should be considered fixed.

\subsubsection{Unrestricted mixed model}

Generally, when one of the main factors (treatment or environment) is fixed, the sum of the interaction effects in relation to the fixed factor is assumed to be zero. In the unrestricted mixed model, the elements of the covariance matrix of $\mathrm{Y}$ are:

$$
\begin{aligned}
& \mathrm{C}_{0}=\sigma_{{ }_{\mathrm{t}}}^{2}+\sigma_{\mathrm{r}}^{2}+\sigma_{\mathrm{b}}^{2}+\sigma_{\mathrm{ta}}^{2}+\sigma^{2} \\
& \mathrm{C}_{1}=\sigma_{\mathrm{r}}^{2}+\sigma_{\mathrm{b}}^{2} \\
& \mathrm{C}_{2}=\sigma_{\mathrm{r}}^{2} \\
& \mathrm{C}_{3}=\sigma^{2}{ }_{\mathrm{t}}+\sigma^{2}{ }_{\mathrm{ta}}
\end{aligned}
$$

$$
\begin{aligned}
& \mathrm{C}_{4}=0 \\
& \mathrm{C}_{5}=\sigma^{2}{ }_{t} \\
& \mathrm{C}_{6}=0
\end{aligned}
$$

Considering the expectation of quadratic forms, the following expected values and the expected mean squares of the analyses of variance can be demonstrated:

$$
\begin{aligned}
& \mathrm{E}\left(\mathrm{Y}^{\prime} \mathrm{Y}\right)=\mathrm{n} \mu^{2}+\mathrm{n} \sigma_{\mathrm{t}}^{2}+\mathrm{n} \sigma_{\mathrm{r}}^{2}+\mathrm{n} \sigma_{\mathrm{b}}^{2}+\mathrm{mv} \sum_{\mathrm{g}=1}^{\mathrm{s}} \mathrm{a}_{\mathrm{g}}^{2}+\mathrm{n} \sigma_{\mathrm{ta}}^{2} \\
& +\mathrm{n} \sigma^{2}+2 \mathrm{mv} \mu \sum_{\mathrm{g}=1}^{\mathrm{s}} \mathrm{a}_{\mathrm{g}}
\end{aligned}
$$$$
\begin{aligned}
& \mathrm{E}[\mathrm{R}(\mu)]=\mathrm{n} \mu^{2}+\mathrm{ms}_{\mathrm{s}}^{2}{ }_{\mathrm{t}}+\mathrm{v}{\sigma_{\mathrm{r}}^{2}}^{2}+\mathrm{k} \sigma_{\mathrm{b}}^{2}+\frac{\mathrm{mv}}{\mathrm{s}}\left(\sum_{\mathrm{g}=1}^{\mathrm{s}} \mathrm{a}_{\mathrm{g}}\right)^{2}+ \\
& \mathrm{m} \sigma_{\mathrm{ta}}^{2}+\sigma^{2}+2 \mathrm{mv} \mu \sum_{\mathrm{g}=1}^{\mathrm{s}} \mathrm{a}_{\mathrm{g}}
\end{aligned}
$$

$\mathrm{E}[\mathrm{R}(\mu, \tau, \delta, \alpha, \beta)]=\mathrm{n} \mu^{2}+\mathrm{n} \sigma_{\mathrm{t}}^{2}+\mathrm{n} \sigma_{\mathrm{r}}^{2}+\mathrm{n} \sigma_{\mathrm{b}}^{2}+\mathrm{mv} \sum_{\mathrm{g}=1}^{\mathrm{s}} \mathrm{a}_{\mathrm{g}}^{2}+$ $[\mathrm{mks}+\mathrm{mk}(\mathrm{k}-1)] \sigma_{\mathrm{ta}}^{2}+(\mathrm{v}+\mathrm{mks}-1) \sigma^{2}+2 \mathrm{mv} \mu \sum_{\mathrm{g}=1}^{\mathrm{s}} \mathrm{a}_{\mathrm{g}}$ 
Table 1. Analyses of variance of group of square lattice experiments

\begin{tabular}{|c|c|c|c|}
\hline Source of variation & Degrees of freedom & Sum of squares & Mean square \\
\hline 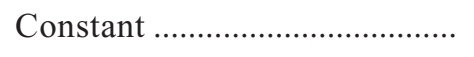 & 1 & $\mathrm{R}(\mu)$ & $\mathrm{R}(\mu)$ \\
\hline Replications|Environment ...... & $\mathrm{s}(\mathrm{m}-1)$ & $\mathrm{R}(\alpha \mid \mu, \delta)$ & MSR $\mid \mathrm{E}$ \\
\hline Blocks|Rep.|Env. ........................... & $\operatorname{sm}(\mathrm{k}-1)$ & $\mathrm{R}(\beta \mid \mu, \delta, \alpha)$ & $\mathrm{MSB}|\mathrm{R}| \mathrm{E}$ (N.Adj.) \\
\hline Treatments (Adjusted) .............. & $(\mathrm{v}-1)$ & $\mathrm{R}(\tau \mid \mu, \delta, \alpha, \beta)$ & MST(Adj.) \\
\hline 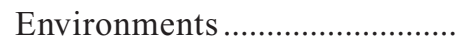 & $(s-1)$ & $\mathrm{R}(\delta \mid \mu)$ & MSE \\
\hline Treatments x Environments ... & $(\mathrm{v}-1)(\mathrm{s}-1)$ & $\mathrm{R}(\tau \delta \mid \mu, \tau, \delta, \alpha, \beta)$ & MSTE \\
\hline Error ……........................................ & $\mathrm{s}(\mathrm{k}-1)(\mathrm{mk}-\mathrm{k}-1)$ & $\mathrm{Y}^{\prime} \mathrm{Y}-\mathrm{R}(\mu, \tau, \alpha, \beta, \delta, \tau \delta)$ & MSe \\
\hline 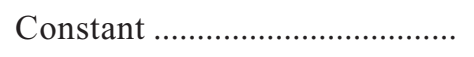 & 1 & $\mathrm{R}(\mu)$ & $\mathrm{R}(\mu)$ \\
\hline Replications|Environment ....... & $\mathrm{s}(\mathrm{m}-1)$ & $\mathrm{R}(\alpha \mid \mu, \delta)$ & $\mathrm{MSR} \mid \mathrm{E}$ \\
\hline Blocks|Rep.|Env. (Adjusted) .. & $\operatorname{sm}(\mathrm{k}-1)$ & $\mathrm{R}(\beta \mid \mu, \tau, \delta, \alpha)$ & $\mathrm{MSB}|\mathrm{R}| \mathrm{E}(\mathrm{Adj})$. \\
\hline 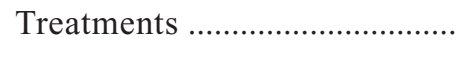 & $(v-1)$ & $\mathrm{R}(\tau \mid \mu, \delta, \alpha)$ & MST(N.Adj.) \\
\hline 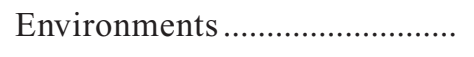 & $(\mathrm{s}-1)$ & $\mathrm{R}(\delta \mid \mu)$ & MSE \\
\hline Treatments x Environments ... & $(v-1)(s-1)$ & $\mathrm{R}(\tau \delta \mid \mu, \tau, \delta, \alpha, \beta)$ & MSTE \\
\hline Error & $\mathrm{s}(\mathrm{k}-1)(\mathrm{mk}-\mathrm{k}-1)$ & $\mathrm{Y}^{\prime} \mathrm{Y}-\mathrm{R}(\mu, \tau, \alpha, \beta, \delta, \tau \delta)$ & MSe \\
\hline
\end{tabular}

Table 2. Expected mean squares of the joint analyses of variance of square lattices, considering the random model

\begin{tabular}{|c|c|}
\hline Source of variation & E(M.S.) \\
\hline Replications|Environment .................... & $\sigma^{2}+\mathrm{k} \sigma_{\mathrm{b}}^{2}+\mathrm{v} \sigma_{\mathrm{b}}^{2}$ \\
\hline 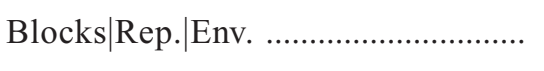 & $\sigma^{2}+\sigma_{\mathrm{ta}}^{2}+\sigma_{\mathrm{t}}^{2}+\mathrm{k} \sigma_{\mathrm{b}}^{2}$ \\
\hline 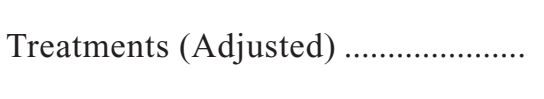 & $\sigma^{2}+\left(\frac{\mathrm{k}}{\mathrm{k}+1}\right) \mathrm{m}^{2}{ }_{\mathrm{ta}}+\left(\frac{\mathrm{k}}{\mathrm{k}+1}\right) \mathrm{ms}^{2}{ }_{\mathrm{t}}$ \\
\hline 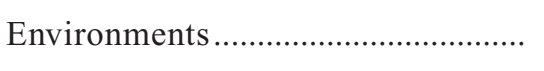 & $\sigma^{2}+\mathrm{m}_{\mathrm{ta}}^{2}+\mathrm{v} \sigma_{\mathrm{r}}^{2}+\mathrm{k} \sigma_{\mathrm{b}}^{2}+\mathrm{mv}_{\mathrm{a}}^{2}$ \\
\hline Treatments x Environments ............... & $\sigma^{2}+\left(\frac{\mathrm{k}}{\mathrm{k}+1}\right) \mathrm{m} \sigma_{\mathrm{ta}}^{2}$ \\
\hline 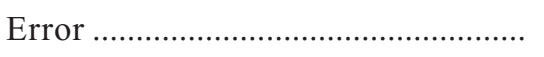 & $\sigma^{2}$ \\
\hline Replications|Environment .................. & $\sigma^{2}+\mathrm{k} \sigma_{\mathrm{b}}^{2}+\mathrm{v} \sigma_{\mathrm{r}}^{2}$ \\
\hline Blocks|Rep.|Env. (Adjusted) ............ & $\sigma^{2}+\left(\frac{\mathrm{s}-1}{\mathrm{~s}}\right) \sigma_{\mathrm{ta}}^{2}+\left(\frac{\mathrm{ms}-1}{\mathrm{~ms}}\right) \mathrm{k} \sigma_{\mathrm{b}}^{2}$ \\
\hline 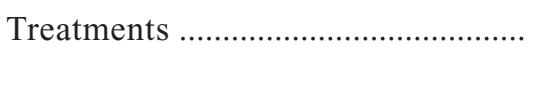 & $\sigma^{2}+m \sigma_{t a}^{2}+\left(\frac{k}{k+1}\right) \sigma_{b}^{2}+m s \sigma_{t}^{2}$ \\
\hline 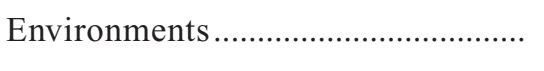 & $\sigma^{2}+m \sigma_{t a}^{2}+v \sigma_{r}^{2}+k \sigma_{b}^{2}+m v \sigma_{a}^{2}$ \\
\hline Treatments x Environments .............. & $\sigma^{2}+\left(\frac{\mathrm{k}}{\mathrm{k}+1}\right) \mathrm{m}_{\mathrm{ta}}^{2}$ \\
\hline 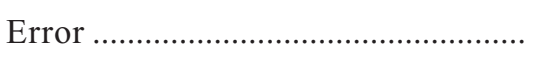 & $\sigma^{2}$ \\
\hline
\end{tabular}


Table 3. Expected mean squares of the joint analyses of variance of square lattices, considering random model, in relation to the variable $\mathrm{Y}+\mathrm{X}$

\begin{tabular}{|c|c|}
\hline Source of variation & E(M.S.) \\
\hline Replications|Env. & {$\left[\sigma_{\mathrm{Y}}^{2}+\mathrm{k} \sigma_{\mathrm{bY}}^{2}+\mathrm{v} \sigma_{\mathrm{rY}}^{2}\right]+\left[\sigma_{\mathrm{X}}^{2}+\mathrm{k} \sigma_{\mathrm{bX}}^{2}+\mathrm{v} \sigma_{\mathrm{rX}}^{2}\right]+2\left[\sigma_{\mathrm{YX}}+\mathrm{k} \sigma_{\mathrm{bYX}}+\mathrm{v} \sigma_{\mathrm{rYX}}\right]$} \\
\hline Blocks $|\mathrm{R}| \mathrm{E}$ & {$\left[\sigma_{\mathrm{Y}}^{2}+\sigma_{\mathrm{taY}}^{2}+\sigma_{\mathrm{tY}}^{2}+\mathrm{k} \sigma_{\mathrm{bY}}^{2}\right]+\left[\sigma_{\mathrm{X}}^{2}+\sigma_{\mathrm{taX}}^{2}+\sigma_{\mathrm{tX}}^{2}+\mathrm{k} \sigma_{\mathrm{bX}}^{2}\right]+2\left[\sigma_{\mathrm{YX}}+\sigma_{\mathrm{taYX}}+\sigma_{\mathrm{tYX}}+\mathrm{k} \sigma_{\mathrm{bYX}}\right]$} \\
\hline Treatments (Adj.) & {$\left[\sigma_{\mathrm{Y}}^{2}+\left(\frac{\mathrm{k}}{\mathrm{k}+1}\right) \mathrm{m}_{\mathrm{taY}}^{2}+\left(\frac{\mathrm{k}}{\mathrm{k}+1}\right) \mathrm{ms} \sigma_{\mathrm{tY}}^{2}\right]+\left[\sigma_{\mathrm{X}}^{2}+\left(\frac{\mathrm{k}}{\mathrm{k}+1}\right) \mathrm{m}_{\mathrm{taX}}^{2}+\left(\frac{\mathrm{k}}{\mathrm{k}+1}\right) \mathrm{ms}_{\mathrm{tX}}^{2}\right]+2\left[\sigma_{\mathrm{YX}}+\left(\frac{\mathrm{k}}{\mathrm{k}+1}\right) \mathrm{m} \sigma_{\mathrm{taYX}}+\left(\frac{\mathrm{k}}{\mathrm{k}+1}\right) \mathrm{ms} \sigma_{\mathrm{tYX}}\right]$} \\
\hline Environments & {$\left[\sigma_{\mathrm{Y}}^{2}+\mathrm{m} \sigma_{\mathrm{taY}}^{2}+\mathrm{v} \sigma_{\mathrm{rY}}^{2}+\mathrm{k} \sigma_{\mathrm{bY}}^{2}+\mathrm{mv}^{2} \sigma_{\mathrm{aY}}\right]+\left[\sigma_{\mathrm{X}}^{2}+\mathrm{m}_{\mathrm{taX}}^{2}+\mathrm{v} \sigma_{\mathrm{rX}}^{2}+\mathrm{k} \sigma_{\mathrm{bX}}^{2}+\mathrm{mv}^{2} \sigma_{\mathrm{aX}}\right]+2\left[\sigma_{\mathrm{YX}}+\mathrm{m}_{\mathrm{taYX}}+\mathrm{v} \sigma_{\mathrm{rYX}}+\mathrm{k} \sigma_{\mathrm{bYX}}+\mathrm{mv} \sigma_{\mathrm{aYX}}\right]$} \\
\hline Treat. x Env. & {$\left[\sigma_{\mathrm{Y}}^{2}+\left(\frac{\mathrm{k}}{\mathrm{k}+1}\right) \mathrm{m} \sigma_{\mathrm{taY}}^{2}\right]+\left[\sigma_{\mathrm{X}}^{2}+\left(\frac{\mathrm{k}}{\mathrm{k}+1}\right) \mathrm{m} \sigma_{\mathrm{taX}}^{2}\right]+2\left[\sigma_{\mathrm{YX}}+\left(\frac{\mathrm{k}}{\mathrm{k}+1}\right) \mathrm{m} \sigma_{\mathrm{taYX}}\right]$} \\
\hline Error & {$\left[\sigma_{\mathrm{Y}}^{2}+\sigma_{\mathrm{X}}^{2}+2 \sigma_{\mathrm{YX}}\right]$} \\
\hline Replications|Env. & {$\left[\sigma_{\mathrm{Y}}^{2}+\mathrm{k} \sigma_{\mathrm{bY}}^{2}+\mathrm{v} \sigma_{\mathrm{rY}}^{2}\right]+\left[\sigma_{\mathrm{X}}^{2}+\mathrm{k} \sigma_{\mathrm{bX}}^{2}+\mathrm{v} \sigma_{\mathrm{rX}}^{2}\right]+2\left[\sigma_{\mathrm{YX}}+\mathrm{k} \sigma_{\mathrm{bYX}}+\mathrm{v} \sigma_{\mathrm{rYX}}\right]$} \\
\hline Blocks $|\mathrm{R}| \mathrm{E}$ (Adj.) & {$\left[\sigma_{\mathrm{Y}}^{2}+\left(\frac{\mathrm{s}-1}{\mathrm{~s}}\right) \sigma_{\mathrm{taY}}^{2}+\left(\frac{\mathrm{ms}-1}{\mathrm{~ms}}\right) \mathrm{k} \sigma_{\mathrm{bY}}^{2}\right]+\left[\sigma_{\mathrm{X}}^{2}+\left(\frac{\mathrm{s}-1}{\mathrm{~s}}\right) \sigma_{\mathrm{taX}}^{2}+\left(\frac{\mathrm{ms}-1}{\mathrm{~ms}}\right) \mathrm{k} \sigma_{\mathrm{bX}}^{2}\right]+2\left[\sigma_{\mathrm{YX}}+\left(\frac{\mathrm{s}-1}{\mathrm{~s}}\right) \sigma_{\mathrm{taYX}}+\left(\frac{\mathrm{ms}-1}{\mathrm{~ms}}\right) \mathrm{k} \sigma_{\mathrm{bYX}}\right]$} \\
\hline Treatments & {$\left[\sigma_{\mathrm{Y}}^{2}+\mathrm{m} \sigma_{\mathrm{taY}}^{2}+\left(\frac{\mathrm{k}}{\mathrm{k}+1}\right) \sigma_{\mathrm{bY}}^{2}+\mathrm{ms} \sigma_{\mathrm{tY}}^{2}\right]+\left[\sigma_{\mathrm{X}}^{2}+\mathrm{m}_{\mathrm{taX}}^{2}+\left(\frac{\mathrm{k}}{\mathrm{k}+1}\right) \sigma_{\mathrm{bX}}^{2}+\mathrm{ms}_{\mathrm{tX}}^{2}\right]+2\left[\sigma_{\mathrm{YX}}+\mathrm{m} \sigma_{\mathrm{taYX}}+\left(\frac{\mathrm{k}}{\mathrm{k}+1}\right) \sigma_{\mathrm{bYX}}+\mathrm{ms}_{\mathrm{tYX}}\right]$} \\
\hline Environments & {$\left[\sigma_{\mathrm{Y}}^{2}+\mathrm{m} \sigma_{\mathrm{taY}}^{2}+\mathrm{v} \sigma_{\mathrm{rY}}^{2}+\mathrm{k} \sigma_{\mathrm{bY}}^{2}+\mathrm{mv}^{2} \sigma_{\mathrm{aY}}\right]+\left[\sigma_{\mathrm{X}}^{2}+\mathrm{m}_{\mathrm{taX}}^{2}+\mathrm{v} \sigma_{\mathrm{rX}}^{2}+\mathrm{k} \sigma_{\mathrm{bX}}^{2}+\mathrm{mv}^{2}{ }_{\mathrm{aX}}\right]+2\left[\sigma_{\mathrm{YX}}+\mathrm{m} \sigma_{\mathrm{taYX}}+\mathrm{v} \sigma_{\mathrm{rYX}}+\mathrm{k} \sigma_{\mathrm{bYX}}+\mathrm{mv} \sigma_{\mathrm{aYX}}\right]$} \\
\hline Treat. x Env. & {$\left[\sigma_{\mathrm{Y}}^{2}+\left(\frac{\mathrm{k}}{\mathrm{k}+1}\right) \mathrm{m} \sigma_{\mathrm{taY}}^{2}\right]+\left[\sigma_{\mathrm{X}}^{2}+\left(\frac{\mathrm{k}}{\mathrm{k}+1}\right) \mathrm{m} \sigma_{\mathrm{taX}}^{2}\right]+2\left[\sigma_{\mathrm{YX}}+\left(\frac{\mathrm{k}}{\mathrm{k}+1}\right) \mathrm{m \sigma}_{\mathrm{taYX}}\right]$} \\
\hline Error & {$\left[\sigma_{Y}^{2}+\sigma_{X}^{2}+2 \sigma_{Y X}\right]$} \\
\hline
\end{tabular}


The expected mean squares are identical to those presented for the random model, with

$\phi a=\left(\frac{1}{s-1}\right)\left[\sum_{g=1}^{s} a_{g}^{2}-\frac{\left(\sum_{g=1}^{s} a_{g}\right)^{2}}{s}\right]=\left(\frac{1}{s-1}\right) \sum_{g=1}^{s}\left[a_{g}-\frac{\sum_{g=1}^{s} a_{g}}{s}\right]^{2}=$

$=\left(\frac{1}{\mathrm{~s}-1}\right) \sum_{\mathrm{g}=1}^{\mathrm{s}}\left(\mathrm{a}_{\mathrm{g}}-\overline{\mathrm{a}}\right)^{2}$

in the place of $\sigma_{\mathrm{a}}^{2}$. Therefore, the estimators of the component $\sigma_{\mathrm{t}}^{2}$ are identical to those of the random model.

As seen, the statistical models (1), (2) and (3) are considered to estimate the covariance component due to treatment effect $\left(\sigma_{\mathrm{tYX}}\right)$, with $\mathrm{a}_{\mathrm{gY}}, \mathrm{a}_{\mathrm{gX}}$ and $\mathrm{a}_{\mathrm{g}}=$ $\mathrm{a}_{\mathrm{gY}}+\mathrm{a}_{\mathrm{gX}}$ as fixed effects. Using previous results and since

$\phi_{a}=\left(\frac{1}{S-1}\right)\left[\sum_{g=1}^{s}\left(a_{g Y}+a_{g X}\right)^{2}-\frac{\left[\sum_{g=1}^{s} a_{g Y}+a_{g X}\right]^{2}}{s}\right]=$

$\left(\frac{1}{S-1}\right)\left[\sum_{g=1}^{s} a_{g Y}^{2}-\frac{\left(\sum_{g=1}^{s} a_{g Y}\right)^{2}}{s}\right]+\left(\frac{1}{s-1}\right)\left[\sum_{g=1}^{s} a_{g X}^{2}-\frac{\left(\sum_{g=1}^{s} a_{g X}\right)^{2}}{s}\right]$

$+2\left(\frac{1}{\mathrm{~s}-1}\right)\left[\sum_{\mathrm{g}=1}^{\mathrm{s}}\left(\mathrm{a}_{\mathrm{gY}} \mathrm{a}_{\mathrm{gX}}\right)-\frac{\left(\sum_{\mathrm{g}=1}^{\mathrm{s}} \mathrm{a}_{\mathrm{gY}}\right)\left(\sum_{\mathrm{g}=1}^{\mathrm{s}} \mathrm{a}_{\mathrm{gX}}\right)^{2}}{\mathrm{~s}}\right]=$

$=\phi_{\mathrm{aY}}+\phi_{\mathrm{aX}}+2 \phi_{\mathrm{aYX}}$

the expected mean squares of the analyses of variance of the variable $\mathrm{Y}+\mathrm{X}$ are demonstrated. The expected mean squares are identical to those presented for the random model, with $\phi_{\mathrm{aY}}, \phi_{\mathrm{aX}}$ and $\phi_{\mathrm{aYX}}$ in the place of $\sigma_{\text {aY }}^{2}, \sigma_{\text {aX }}^{2}$ and $\sigma_{\text {aYX }}$, respectively. Therefore, the estimators of $\sigma_{\mathrm{TYX}}$ are equal to those of the random model.

\subsubsection{Restricted mixed model}

In the mixed model with the restriction

$$
\sum_{\mathrm{g}=1}^{\mathrm{s}}(\mathrm{ta})_{\mathrm{ig}}=0 \text {, to all } \mathrm{i},
$$

not all effects of interaction between treatment and environment are independent random variables.

In this model:

$\mathrm{V}\left[\sum_{\mathrm{g}=1}^{\mathrm{s}}(\mathrm{ta})_{\mathrm{ig}}\right]=\mathrm{s} \sigma_{\mathrm{ta}}^{2}+2 \sum_{\mathrm{g}=1}^{\mathrm{s}} \sum_{\mathrm{g}=1}^{\mathrm{s}} \operatorname{Cov}\left[(\mathrm{ta})_{\mathrm{ig}},(\mathrm{ta})_{\mathrm{ig}}\right]=0$,

assuming that $\operatorname{Cov}\left[(\mathrm{ta})_{\mathrm{ig}},(\mathrm{ta})_{\mathrm{ig}}\right]=\mathrm{E}\left[(\mathrm{ta})_{\mathrm{ig}}(\mathrm{ta})_{\mathrm{ig}}\right]=\sigma$, to all $\mathrm{i}, \mathrm{g}$ and $\mathrm{g}^{\prime}\left(\mathrm{g} \cdot \mathrm{g}{ }^{\prime}\right)$, we have:

$$
\sigma=-\left(\frac{1}{\mathrm{~s}-1}\right) \sigma_{\mathrm{ta}}^{2}
$$

Therefore, in the matrix $\Sigma$,

$$
\begin{aligned}
& \mathrm{C}_{0}=\sigma_{\mathrm{t}}^{2}+\sigma_{\mathrm{r}}^{2}+\sigma_{\mathrm{b}}^{2}+\sigma_{\mathrm{ta}}^{2}+\sigma^{2} \\
& \mathrm{C}_{1}=\sigma_{\mathrm{r}}^{2}+\sigma_{\mathrm{b}}^{2} \\
& \mathrm{C}_{2}=\sigma_{\mathrm{r}}^{2} \\
& \mathrm{C}_{3}=\sigma_{{ }_{\mathrm{t}}}+\sigma_{{ }_{\mathrm{ta}}}^{2} \\
& \mathrm{C}_{4}=0 \\
& \mathrm{C}_{5}=\sigma_{\mathrm{t}}^{2}-\left(\frac{1}{\mathrm{~s}-1}\right) \sigma_{\mathrm{ta}}^{2} \\
& \mathrm{C}_{6}=0
\end{aligned}
$$

Using the expectation of quadratic forms, the expected values below and those presented in Table 4 can be obtained:

$$
\begin{aligned}
& \mathrm{E}\left(\mathrm{Y}^{\prime} \mathrm{Y}\right)=\mathrm{n} \mu^{2}+\mathrm{n}{\sigma^{2}}_{\mathrm{t}}+\mathrm{ns}_{\mathrm{s}}{ }_{\mathrm{r}}^{2}+\mathrm{n} \sigma_{\mathrm{b}}^{2}+\mathrm{mv} \sum_{\mathrm{g}=1}^{\mathrm{s}} \mathrm{a}_{\mathrm{g}}^{2}+\mathrm{n}{\sigma^{2}}_{\mathrm{ta}}+ \\
& \mathrm{n} \sigma^{2}+2 \mathrm{mv} \mu \sum_{\mathrm{g}=1 \mathrm{~g}}^{\mathrm{s}} \mathrm{a}_{\mathrm{g}} \\
& \begin{aligned}
\mathrm{E}[\mathrm{R}(\mu)] & =\mathrm{n} \mu+\operatorname{ms}_{\mathrm{s}}{\sigma_{\mathrm{t}}^{2}}_{\mathrm{t}}+\mathrm{v}{\sigma_{\mathrm{r}}^{2}}^{2}+\mathrm{k} \sigma_{\mathrm{b}}^{2}+\frac{\mathrm{mv}}{\mathrm{s}}\left(\sum_{\mathrm{g}=1}^{\mathrm{s}} \mathrm{a}_{\mathrm{g}}\right)^{2}+\sigma^{2} \\
& +2 \mathrm{mv} \mu \sum_{\mathrm{g}=1} \mathrm{a}_{\mathrm{g}}
\end{aligned}
\end{aligned}
$$

$\mathrm{E}[\mathrm{R}(\mu, \tau, \delta, \alpha, \beta)]=n \mu^{2}+\mathrm{n} \sigma_{\mathrm{t}}^{2}+\mathrm{n} \sigma_{\mathrm{r}}^{2}+\mathrm{n} \sigma_{\mathrm{b}}^{2}+\mathrm{mv} \sum_{\mathrm{g}=1}^{\mathrm{s}} \mathrm{a}_{\mathrm{g}}{ }_{\mathrm{g}}$ $+\mathrm{mks}_{\mathrm{ta}}^{2}+(\mathrm{v}+\mathrm{mks}-1) \sigma^{2}+2 \mathrm{mv} \mu \sum_{\mathrm{g}=1}^{\mathrm{s}} \mathrm{a}_{\mathrm{g}}$

The estimators of $\sigma_{t}^{2}$ are: 


$$
\begin{aligned}
& \hat{\sigma}_{t(1)}^{2}=\left(\frac{k+1}{k}\right)\left[\frac{M S T(A d j .)-M S e}{m s}\right] \\
& \hat{\sigma}_{\mathrm{t}(2)}^{2}=\left[\frac{\operatorname{MST}(\mathrm{N} . A d j .)-\mathrm{MSe}-\left(\frac{\mathrm{k}}{\mathrm{k}+1}\right) \hat{\sigma}_{\mathrm{b}(2)}^{2}}{\mathrm{~ms}}\right] \neq\left[\frac{\mathrm{MST}(\mathrm{N} . \text { Adj. })-\mathrm{MSe}_{\mathrm{CB}}}{\mathrm{ms}}\right]
\end{aligned}
$$

where:

$$
\left.\left.\hat{\sigma}_{\mathrm{b}(2)}^{2}=\left[\frac{\mathrm{ms}}{\mathrm{k}(\mathrm{ms}-1)}\right][\mathrm{MSB}\} \mathrm{R}\right\} \mathrm{E}(\operatorname{Adj} .)-\mathrm{MSe}-\left(\frac{\mathrm{s}-1}{\mathrm{~s}}\right)\left(\frac{\mathrm{k}+1}{\mathrm{k}}\right)\left(\frac{\mathrm{MST}-\mathrm{MSe}}{\mathrm{m}}\right)\right]
$$

and $\mathrm{MSe}_{\mathrm{CB}}$ is the error mean square, considering the complete block model. Therefore, $\hat{\sigma}_{\mathrm{t}(2)}^{2}$ is not the estimator of $\sigma^{2}$ of the analysis according to the complete block model.

The statistical models (1), (2) and (3) are adjusted to estimate the covariance component $\sigma_{\mathrm{tYX}}$.
Based on previous results and on the assumptions about the restricted mixed model, and since not all effects $(\mathrm{ta})_{\mathrm{ig}}=(\mathrm{ta})_{\mathrm{igY}}+(\mathrm{ta})_{\mathrm{igx}}$ are independent, the expected mean squares presented in Table 5 are demonstrated. The estimators of $\sigma_{\mathrm{tYX}}$ are:

\begin{tabular}{|c|c|}
\hline Source of variation & E(M.S.) \\
\hline Replications|Environment ................. & $\sigma^{2}+\mathrm{k} \sigma_{\mathrm{b}}^{2}+\mathrm{v} \sigma_{\mathrm{b}}^{2}$ \\
\hline 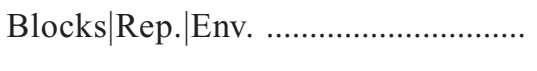 & $\sigma^{2}+\sigma_{{ }_{\mathrm{ta}}}^{2}+\sigma_{\mathrm{t}}^{2}+\mathrm{k} \sigma_{\mathrm{b}}^{2}$ \\
\hline Treatments (Adjusted) ........................... & $\sigma^{2}+\left(\frac{\mathrm{k}}{\mathrm{k}+1}\right) \mathrm{ms}_{\mathrm{t}}^{2}$ \\
\hline 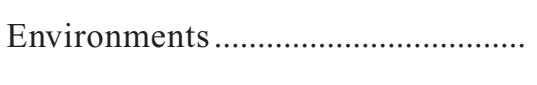 & $\sigma^{2}+\left(\frac{\mathrm{S}}{\mathrm{s}-1}\right) \mathrm{m} \sigma_{\mathrm{ta}}^{2}+\mathrm{v} \sigma_{\mathrm{r}}^{2}+\mathrm{k} \sigma_{\mathrm{b}}^{2}+\mathrm{mv}^{2}{ }_{\mathrm{a}}^{2}$ \\
\hline Treatments x Environments ............. & $\sigma^{2}+\left(\frac{\mathrm{s}}{\mathrm{S}-1}\right)\left(\frac{\mathrm{k}}{\mathrm{k}+1}\right) \mathrm{m}_{\mathrm{ta}}^{2}$ \\
\hline 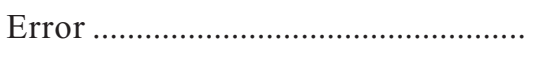 & $\sigma^{2}$ \\
\hline Replications|Environment ................... & $\sigma^{2}+\mathrm{k} \sigma_{\mathrm{b}}^{2}+\mathrm{v} \sigma_{\mathrm{r}}^{2}$ \\
\hline Blocks|Rep.|Env. (Adjusted) ............. & $\sigma^{2}+\sigma_{\mathrm{ta}}^{2}+\left(\frac{\mathrm{ms}-1}{\mathrm{~ms}}\right) \mathrm{k} \sigma_{\mathrm{b}}^{2}$ \\
\hline 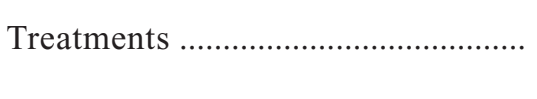 & $\sigma^{2}+\left(\frac{\mathrm{k}}{\mathrm{k}+1}\right) \sigma_{\mathrm{b}}^{2}+\mathrm{ms}_{\mathrm{t}}^{2}$ \\
\hline 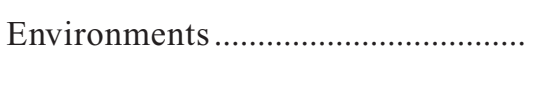 & $\sigma^{2}+\left(\frac{\mathrm{s}}{\mathrm{s}-1}\right) \mathrm{m} \sigma_{\mathrm{ta}}^{2}+\mathrm{v} \sigma_{\mathrm{r}}^{2}+\mathrm{k} \sigma_{\mathrm{b}}^{2}+\mathrm{mv} \phi_{\mathrm{a}}$ \\
\hline Treatments x Environments ................ & $\sigma^{2}+\left(\frac{\mathrm{s}}{\mathrm{s}-1}\right)\left(\frac{\mathrm{k}}{\mathrm{k}+1}\right) \mathrm{m} \sigma_{\mathrm{ta}}^{2}$ \\
\hline 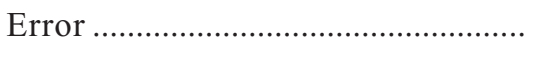 & $\sigma^{2}$ \\
\hline
\end{tabular}

Table 4. Expected mean squares of the joint analyses of variance of square lattices, considering restricted mixed model ${ }^{1}$ with environment effect fixed and other effects random

${ }^{(1)}$ Under the restrictions $\sum_{\mathrm{g}=1}^{\mathrm{s}}(\mathrm{ta})_{\mathrm{ig}}=0$, to all i. 
$\hat{\sigma}_{\mathrm{tYX}(1)}=\left(\frac{\mathrm{k}+1}{\mathrm{k}}\right)\left\{\frac{[\operatorname{MST}(\operatorname{Adj} .)(\mathrm{Y}+\mathrm{X})-\operatorname{MST}(\operatorname{Adj} .)(\mathrm{Y})-\operatorname{MST}(\operatorname{Adj} .)(\mathrm{X})]-[\operatorname{MSe}(\mathrm{Y}+\mathrm{X})-\operatorname{MSe}(\mathrm{Y})-\operatorname{MSe}(\mathrm{X})]}{2 \mathrm{~ms}}\right\}$

$\hat{\sigma}_{\mathrm{tYX}(2)}=\left[\frac{\operatorname{MST}(\mathrm{N} . A d j .)(\mathrm{Y}+\mathrm{X})-\operatorname{MST}(\mathrm{N} \cdot \operatorname{Adj} .)(\mathrm{Y})-\operatorname{MST}(\mathrm{N} . A d j .)(\mathrm{X})-2 \hat{\sigma}_{\mathrm{YX}}-2\left(\frac{\mathrm{k}}{\mathrm{k}+1}\right) \hat{\sigma}_{\mathrm{bYX}(2)}}{2 \mathrm{~ms}}\right]$

where:

$\hat{\sigma}_{\mathrm{YX}}=\frac{\operatorname{MSe}(\mathrm{Y}+\mathrm{X})-\operatorname{MSe}(\mathrm{Y})-\operatorname{MSe}(\mathrm{X})}{2}$

$\hat{\sigma}_{\mathrm{bYX}(2)}=\left[\frac{\mathrm{ms}}{2 \mathrm{k}(\mathrm{ms}-1)}\right]\left[\mathrm{MSB} \neq \mathrm{R} \neq \mathrm{E}(\operatorname{Adj}).(\mathrm{Y}+\mathrm{X})-\mathrm{MSB} \neq \mathrm{R} \neq \mathrm{E}(\operatorname{Adj}).(\mathrm{Y})-\mathrm{MSB} \neq \mathrm{R} \neq \mathrm{E}(\operatorname{Adj}).(\mathrm{X})-2 \hat{\sigma}_{\mathrm{YX}}-2 \hat{\sigma}_{\mathrm{taYX}}\right]$

$\hat{\sigma}_{\mathrm{taYX}}=\left(\frac{\mathrm{s}-1}{\mathrm{~s}}\right)\left(\frac{\mathrm{k}+1}{\mathrm{k}}\right)\left(\frac{\operatorname{MSTE}(\mathrm{Y}+\mathrm{X})-\operatorname{MSTE}(\mathrm{Y})-\operatorname{MSTE}(\mathrm{X})-2 \hat{\sigma}_{\mathrm{taYX}}}{2 \mathrm{~m}}\right)$

\section{APPLICATION}

The results of the analyses of variance for height and diameter of 49 half-sib families of Eucalyptus pyrocarpa, from a non inbred population, evaluated in a $7 \times 7$ simple lattice in two different environmental conditions, are shown in Table 6. The SAEG (System for Statistical Analyses) program, developed by the Universidade Federal de Viçosa, was used for the analyses.

Considering unrestricted mixed model, the analyses of variance show, at a level of 5\% of significance, absence of interaction between families and environments, for height and diameter. There is no difference between the means of the reference population in the two environments (two levels of fertilization were used). In the base population there is genetic variability for both characters. As there is evidence of absence of progeny $\mathrm{x}$ environment interaction, selection can be done considering the means of the families in the two levels of fertilization, favoring the choice of those with desired performance in different environments. Estimates of the genotypic variance between progenies, of the covariance between genotypic means of same family, and of some other genetic parameters are presented in Table 7 . The equality between the estimates of $\sigma_{t(1)}^{2}$ and $\sigma_{t(2)}^{2}$ and of $\sigma_{t(1)}$ and $\sigma_{t(2)}$, reveals homogeneity between blocks within replication within environment.

In relation to both characters, the differences between the additive genetic values of the individuals in the base population account for a relevant portion of the variance of the phenotypic means of the families. The magnitude of the two heritabilities indicates that the families with greater phenotypic mean should have a common parent with greater additive genetic value (greater number of genes which increase each trait). The estimates of the correlation between the phenotypic mean of the family and the additive 
Table 5. Expected mean squares of the joint analyses of variance of square lattices, considering restricted mixed model ${ }^{1}$ with $^{\text {environment }}$ effect fixed and other effects random, in relation to the variable $\mathrm{Y}+\mathrm{X}$

\begin{tabular}{|c|c|}
\hline Source of variation & E(M.S.) \\
\hline Replications|Env. & {$\left[\sigma_{\mathrm{Y}}^{2}+\mathrm{k} \sigma_{\mathrm{bY}}^{2}+\mathrm{v} \sigma_{\mathrm{rY}}^{2}\right]+\left[\sigma_{\mathrm{X}}^{2}+\mathrm{k} \sigma_{\mathrm{bX}}^{2}+\mathrm{v} \sigma_{\mathrm{rX}}^{2}\right]+2\left[\sigma_{\mathrm{YX}}+\mathrm{k} \sigma_{\mathrm{bYX}}+\mathrm{v} \sigma_{\mathrm{rYX}}\right]$} \\
\hline Blocks $|\mathrm{R}| \mathrm{E}$ & {$\left[\sigma_{\mathrm{Y}}^{2}+\sigma_{\mathrm{taY}}^{2}+\sigma_{\mathrm{tY}}^{2}+\mathrm{k} \sigma_{\mathrm{bY}}^{2}\right]+\left[\sigma_{\mathrm{X}}^{2}+\sigma_{\mathrm{taX}}^{2}+\sigma_{\mathrm{tX}}^{2}+\mathrm{k} \sigma_{\mathrm{bX}}^{2}\right]+2\left[\sigma_{\mathrm{YX}}+\sigma_{\mathrm{taYX}}+\sigma_{\mathrm{tYX}}+\mathrm{k} \sigma_{\mathrm{bYX}}\right]$} \\
\hline Treatments (Adj.) & {$\left[\sigma_{\mathrm{Y}}^{2}+\left(\frac{\mathrm{k}}{\mathrm{k}+1}\right) \mathrm{ms} \sigma_{\mathrm{tY}}^{2}\right]+\left[\sigma_{\mathrm{X}}^{2}+\left(\frac{\mathrm{k}}{\mathrm{k}+1}\right) \mathrm{ms}_{\mathrm{tX}}^{2}\right]+2\left[\sigma_{\mathrm{YX}}+\left(\frac{\mathrm{k}}{\mathrm{k}+1}\right) \mathrm{ms}_{\mathrm{YX}}^{2}\right]+2\left[\sigma_{\mathrm{YX}}+\left(\frac{\mathrm{k}}{\mathrm{k}+1}\right) \mathrm{ms}_{\mathrm{tYX}}\right]$} \\
\hline Environments & {$\left[\sigma_{\mathrm{Y}}^{2}+\left(\frac{\mathrm{s}}{\mathrm{s}-1}\right) \mathrm{m} \sigma_{\mathrm{taY}}^{2}+\mathrm{v} \sigma_{\mathrm{rY}}^{2}+\mathrm{k} \sigma_{\mathrm{bY}}^{2}+\mathrm{mv}_{\mathrm{aY}}\right]+\left[\sigma_{\mathrm{X}}^{2}+\left(\frac{\mathrm{s}}{\mathrm{s}-1}\right) \mathrm{m \sigma}_{\mathrm{taX}}^{2}+\mathrm{v} \sigma_{\mathrm{rX}}^{2}+\mathrm{k} \sigma_{\mathrm{bX}}^{2}+\mathrm{mv}_{\mathrm{aX}}\right]+2\left[\sigma_{\mathrm{YX}}+\left(\frac{\mathrm{s}}{\mathrm{s}-1}\right) \mathrm{m} \sigma_{\mathrm{taXX}}+\mathrm{v} \sigma_{\mathrm{rYX}}+\mathrm{k} \sigma_{\mathrm{bYX}}+\mathrm{mv}_{\mathrm{aYX}}\right]$} \\
\hline Treat. x Env. & {$\left[\sigma_{\mathrm{Y}}^{2}+\left(\frac{\mathrm{s}}{\mathrm{s}-1}\right)\left(\frac{\mathrm{k}}{\mathrm{k}+1}\right) \mathrm{m} \sigma_{\mathrm{taY}}^{2}\right]+\left[\sigma_{\mathrm{X}}^{2}+\left(\frac{\mathrm{s}}{\mathrm{s}-1}\right)\left(\frac{\mathrm{k}}{\mathrm{k}+1}\right) \mathrm{m} \sigma_{\mathrm{taX}}^{2}\right]+2\left[\sigma_{\mathrm{YX}}+\left(\frac{\mathrm{s}}{\mathrm{s}-1}\right)\left(\frac{\mathrm{k}}{\mathrm{k}+1}\right) \mathrm{m} \sigma_{\mathrm{taYX}}\right]$} \\
\hline Error & $\sigma_{\mathrm{Y}}^{2}+\sigma_{\mathrm{X}}^{2}+2 \sigma_{\mathrm{YX}}$ \\
\hline Replications|Env. & {$\left[\sigma_{\mathrm{Y}}^{2}+\mathrm{k} \sigma_{\mathrm{bY}}^{2}+\mathrm{v} \sigma_{\mathrm{rY}}^{2}\right]+\left[\sigma_{\mathrm{X}}^{2}+\mathrm{k} \sigma_{\mathrm{bX}}^{2}+\mathrm{v} \sigma_{\mathrm{rX}}^{2}\right]+2\left[\sigma_{\mathrm{YX}}+\mathrm{k} \sigma_{\mathrm{bYX}}+\mathrm{v} \sigma_{\mathrm{rYX}}\right]$} \\
\hline Blocks|R|E (Adj.) & {$\left[\sigma_{\mathrm{Y}}^{2}+\sigma_{\mathrm{taY}}^{2}+\left(\frac{\mathrm{ms}-1}{\mathrm{~ms}}\right) \mathrm{k} \sigma_{\mathrm{bY}}^{2}\right]+\left[\sigma_{\mathrm{X}}^{2}+\sigma_{\mathrm{taX}}^{2}+\left(\frac{\mathrm{ms}-1}{\mathrm{~ms}}\right) \mathrm{k} \sigma_{\mathrm{bX}}^{2}\right]+2\left[\sigma_{\mathrm{YX}}+\sigma_{\mathrm{taYX}}+\left(\frac{\mathrm{ms}-1}{\mathrm{~ms}}\right) \mathrm{k} \sigma_{\mathrm{bYX}}\right]$} \\
\hline Treatments & {$\left[\sigma_{\mathrm{Y}}^{2}+\left(\frac{\mathrm{k}}{\mathrm{k}+1}\right) \sigma_{\mathrm{bY}}^{2}+\mathrm{ms}_{\mathrm{tY}}^{2}\right]+\left[\sigma_{\mathrm{X}}^{2}+\left(\frac{\mathrm{k}}{\mathrm{k}+1}\right) \sigma_{\mathrm{bX}}^{2}+\mathrm{ms}_{\mathrm{tX}}^{2}\right]+2\left[\sigma_{\mathrm{YX}}+\left(\frac{\mathrm{k}}{\mathrm{k}+1}\right) \sigma_{\mathrm{bYX}}+\mathrm{ms}_{\mathrm{tYX}}\right]$} \\
\hline Environments & {$\left[\sigma_{\mathrm{Y}}^{2}+\left(\frac{\mathrm{s}}{\mathrm{s}-1}\right) \mathrm{m} \sigma_{\mathrm{taY}}^{2}+\mathrm{v} \sigma_{\mathrm{rY}}^{2}+\mathrm{k} \sigma_{\mathrm{bY}}^{2}+\mathrm{mv}_{\mathrm{aY}}\right]+\left[\sigma_{\mathrm{X}}^{2}+\left(\frac{\mathrm{s}}{\mathrm{s}-1}\right) \mathrm{m}_{\mathrm{taX}}^{2}+\mathrm{v}_{\mathrm{rX}}^{2}+\mathrm{k} \sigma_{\mathrm{bX}}^{2}+\mathrm{mv} \phi_{\mathrm{aX}}\right]+2\left[\sigma_{\mathrm{YX}}+\left(\frac{\mathrm{s}}{\mathrm{s}-1}\right) \mathrm{m} \sigma_{\mathrm{taXX}}+\mathrm{v} \sigma_{\mathrm{rYX}}+\mathrm{k} \sigma_{\mathrm{bYX}}+\mathrm{mv}_{\mathrm{aYX}}\right]$} \\
\hline Treat. x Env. & {$\left[\sigma_{\mathrm{Y}}^{2}+\left(\frac{\mathrm{s}}{\mathrm{s}-1}\right)\left(\frac{\mathrm{k}}{\mathrm{k}+1}\right) \mathrm{m} \sigma_{\mathrm{taY}}^{2}\right]+\left[\sigma_{\mathrm{X}}^{2}+\left(\frac{\mathrm{s}}{\mathrm{s}-1}\right)\left(\frac{\mathrm{k}}{\mathrm{k}+1}\right) \mathrm{m} \sigma_{\mathrm{taX}}^{2}\right]+2\left[\sigma_{\mathrm{YX}}+\left(\frac{\mathrm{s}}{\mathrm{s}-1}\right)\left(\frac{\mathrm{k}}{\mathrm{k}+1}\right) \mathrm{m} \sigma_{\mathrm{taYX}}\right]$} \\
\hline Error & $\sigma_{Y}^{2}+\sigma_{X}^{2}+2 \sigma_{Y X}$ \\
\hline
\end{tabular}


Table 6. Joint analyses of variance of height and diameter of 49 half-sib families of Eucalyptus pyrocarpa

\begin{tabular}{lccc}
\hline \multirow{2}{*}{$\begin{array}{c}\text { Source } \\
\text { of variation }\end{array}$} & $\begin{array}{c}\text { Degrees } \\
\text { of freedom }\end{array}$ & Height & Mean square \\
\cline { 3 - 4 } & & $\mathrm{m}$ & $\mathrm{m}$ \\
Replications|Env. ............... & 2 & 6.7455 & $0.5862 \times 10^{-3}$ \\
Blocks|Rep.|Env. (Adj.) ...... & 24 & 1.9398 & $0.2496 \times 10^{-3}$ \\
Families (Adj.) ................... & 48 & $6.0997^{* *}$ & $0.7704 \times 10^{-3} * *$ \\
Environments ..................... & 1 & $13.0885^{\mathrm{ns}}$ & $0.1046 \times 10^{-2} \mathrm{~ns}$ \\
Families x Environments .... & 48 & $2.3549^{\mathrm{ns}}$ & $0.2987 \times 10^{-3} \mathrm{~ns}$ \\
Error .................................. & 72 & 1.7297 & $0.2148 \times 10^{-3}$ \\
\hline
\end{tabular}

**: $\mathrm{P}<0.01$. ns: not significant at $5 \%$.

Table 7. Estimates of variance and covariance components and of some other genetic parameters ${ }^{1}$, in relation to height and diameter of half-sib families of a population of Eucalyptus pyrocarpa, evaluated in two environments

\begin{tabular}{|c|c|c|}
\hline Parameter & Estimate & Trait \\
\hline & $\mathrm{m}$ & \\
\hline \multirow{2}{*}{ 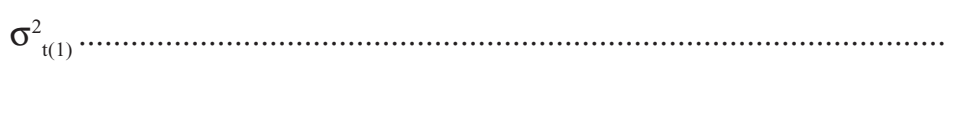 } & Height & 1.07 \\
\hline & Diameter & $0.13 \times 10^{-3}$ \\
\hline \multirow[t]{2}{*}{ 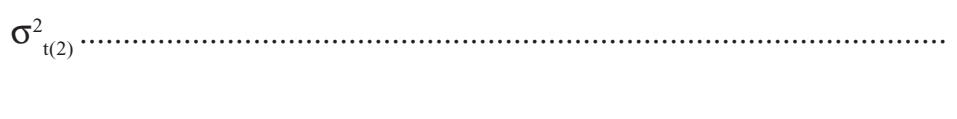 } & Height & 1.03 \\
\hline & Diameter & $0.13 \times 10^{-3}$ \\
\hline$\sigma_{t(1)}$ & - & 0.01 \\
\hline 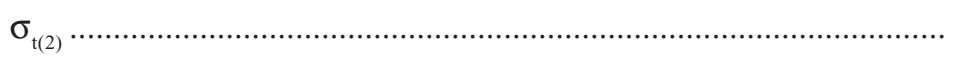 & - & 0.01 \\
\hline \multirow[t]{2}{*}{ 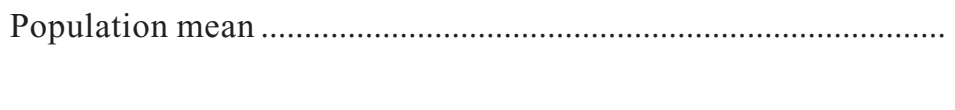 } & Height & 14.02 \\
\hline & Diameter & 0.117 \\
\hline \multirow[t]{2}{*}{ Mean of the selected families (selection based on height) ........... } & Height & 15.49 \\
\hline & Diameter & 0.134 \\
\hline \multirow[t]{2}{*}{ Mean of the selected families (selection based on diameter) ....... } & Height & 15.38 \\
\hline & Diameter & 0.134 \\
\hline \multirow[t]{2}{*}{$\mathrm{h}^{2}$} & Height & 0.61 \\
\hline & Diameter & 0.59 \\
\hline \multirow[t]{2}{*}{ ( $\mathrm{G}$} & Height & $6.58 \%$ \\
\hline & Diameter & $8.52 \%$ \\
\hline $\mathrm{r}_{\mathrm{A}}$ & - & 0.89 \\
\hline
\end{tabular}

${ }^{(1)}$ The values of $\mathrm{h}^{2}$ (heritability in narrow sense, on a family mean basis), $\Delta \mathrm{G}$ (expected genetic gain due to selection and recombination of the 15 best families, expressed as percentage of the mean of the base population) and $\mathrm{r}_{\mathrm{A}}$ (additive genetic correlation between height and diameter), were obtained considering the estimates of $\sigma_{\mathrm{t}(1)}^{2}$ and $\sigma_{\mathrm{t}(1)}$. The variance of the phenotypic means of the families was estimated using the estimator MST(Adj.)/(k/(k+1)) ms. 
genetic value of the common parent (Viana, 1996b) are $\breve{s} \overline{0.61}=0.78$ and $\breve{s} \overline{0.59}=0.77$, for height and diameter, respectively. Thus, the choice of the superior families will alter the genotypic means of height and diameter of the base population, in the desirable direction. In relation to height, the predicted direct genetic gain with selection and recombination of the 15 best families (selection intensity of approximately 1.138 ) is of $6.58 \%$. In relation to diameter, the expected direct gain is $8.52 \%$. The magnitude of the additive genetic correlation between the two traits (Viana, 1996a) shows that the direct selection based on one trait will determine indirect gain in relation to the other. The selection based on height should determine a change in the mean diameter of the base population of $(0.134-0.117)(0.59) .100 / 0.117=8.57 \%$. Evidently, there is no difference between the direct and indirect gains in relation to diameter (because of the equality between direct and indirect selection differentials). With selection considering diameter, the expected indirect gain for height is of $(15.38$ - 14.02) $(0.61) .100 / 14.02=5.92 \%$.

\section{REFERENCES}

ARRIEL, E.F.; PACHECO, C.A.P. \& RAMALHO, M.A.P. Evaluation of maize half-sib families in different plant densities. Pesquisa Agropecuária Brasileira, Brasília, 28(7):849-854, 1993.

BANCROFT, T.A. \& SMITH, A.L. Efficiency of the simple lattice design relative to randomized complete blocks design in cotton variety and strain testing. Agronomy Journal, Madison, 41(4):157-160, 1949.

BENINATI, N.F. \& BUSCH, R.H. Grain protein inheritance and nitrogen uptake and redistribution in a spring wheat cross. Crop Science, Madison, 32(6):1471-1475, 1992.

CHAVES, L.J. \& MIRANDA FILHO, J.B. de. Plot size for progeny selection in maize (Zea mays L.). Theoretical and Applied Genetics, Berlin, 84(7/8):963-970, 1992.

COCHRAN, W.G. \& COX, G.M. Experimental designs. 2.ed. New York, John Wiley \& Sons, 1957. 611p.

FEDERER, W.T. Experimental design: theory and application. New York, Macmillan, 1955. 544p.
FERRÃO, R.G.; GAMA, E.E.G. e; CARVALHO, H.W.L. de \& FERRÃO, M.A.G. Evaluation of the combining ability of twenty maize lines in a partial diallel cross. Pesquisa Agropecuária Brasileira, Brasília, 29(12):1933-1939, 1994.

GRAYBILL, F.A. Theory and application of the linear model. North Scituate, Massachusetts, Duxbury Press, 1976. $704 \mathrm{p}$.

JOHNSON, I.J. \& MURPHY, H.C. Lattice and lattice square designs with oat uniformity data and in variety trials. Journal of The American Society of Agronomy, Washington, 35(4):291-305, 1943.

KEMPTHORNE, O. An introduction to genetic statistics. New York, John Wiley \& Sons, 1957. 545p.

LIN, C.S.; BINNS, M.R.; VOLDENG, H.D. \& GUILLEMETTER, R. Performance of randomized block designs in field experiments. Agronomy Journal, Madison, 85(1):168-171, 1993.

MICHELINI, L.A. \& HALLAUER, A.R. Evaluation of exotic and adapted maize (Zea mays L.) germplasm crosses. Maydica, Bergamo, 38(4): 275-282, 1993.

MONCADA, P.; CASLER, M.D. \& CLAYTON, M.K. An approach to reduce the time required for bean yield evaluation in coffee breeding. Crop Science, Madison, 33(3):448-452, 1993.

OLIVEIRA, A.C. de. Joint analysis of experiments in incomplete block designs with some common treatments - intrablock analysis. Pesquisa Agropecuaria Brasileira, Brasília, 28(11):1255-1262, 1993.

REZENDE, G.D.S.P. \& RAMALHO, M.A.P. Competitive ability of maize and common bean (Phaseolus vulgaris) cultivars intercropped in different environments. Journal of Agricultural Science, London, 123(2):185-190, 1994.

SAHAGUN-CASTELLANOS, J. \& FREY, K.J. Efficiency of three experimental designs for genotype evaluation. Revista Chapingo, Chapingo, 15(71-72):114-122, 1990.

SAS Institute, SAS/STAT ${ }^{\circledR}$. User's guide. Version 6, 4.ed. Cary, NC, SAS Institute, 1989. v.1, 943 p.

SEARLE, S.R. Linear models. New York, John Wiley \& Sons, 1971. 532p.

SEARLE, S.R.; CASELLA, G. \& McCULLOCH, C.E. Variance components. New York, John Wiley \& Sons, 1992. 501p.

SINGH, S.P.; URREA, C.A.; MOLINA, A. \& GUTIERREZ, J.A. Performance of small-seeded common bean from the second selection cycle and multiple-cross intra and interracial populations. Canadian Journal of Plant Science, Ottawa, 72(3):735-741, 1992. 
TORRIE, J.H.; SHANDS, H.L. \& LEITH, B.D. Efficiency studies of types of design with small grain yield trials. Journal of The American Society of Agronomy, Washington, 35(8):645-661, 1943.

VIANA, J.M.S. Correlações entre médias genotípicas de mesma família. In: CONGRESSO NACIONAL DE MILHO E SORGO, 21., Londrina, 1996. Anais. Londrina, Instituto Agronômico do Paraná/Associação Brasileira de Milho e Sorgo, 1996a. p.60.
VIANA, J.M.S. Herdabilidade em nível de média de família. In: CONGRESSO NACIONAL DE MILHO E SORGO, 21., Londrina, 1996. Anais. Londrina, Instituto Agronômico do Paraná/Associação Brasileira de Milho e Sorgo, 1996b. p.61.

WELLHAUSEN, E.J. The accuracy of incomplete block designs in varietal trials in West Virginia. Journal of the American Society of Agronomy, Washington, 35(1):66-76, 1943.

ZUBER, M.S. Relative efficiency of incomplete block designs using corn uniformity trial data. Journal of the American Society of Agronomy, Madison, 34(1):30-47, 1942. 